\title{
0 perspectivismo como fundamento para metodologias de análise das redes sociais: alguns resultados com 0 Twitter
}

\section{Rodrigo Travitzki, Marco Toledo Bastos e Rafael Luís Galdini Raimundo}

\section{Resumo}

Neste artigo, apresentamos uma metodologia de análise das variáveis que influenciam a disseminação da informação na rede social Twitter. Analisamos dados de 595.240 mensagens produzidas por 261.757 usuários e encontramos três variáveis locais relacionadas ao grau de replicação das mensagens (retuíte): 1) o número de respostas recebidas (explicou em média 21\% dos retuítes); 2) número de seguidores na sub-rede (17\%) e 3) o número de tuítes com a hashtag (7\%). Comparando variáveis a priori (globais) e a posteriori (locais), houve diferença significativa no número de seguidores, de mensagens $(p<0,001)$ e também no número de amigos $(p<0,01)$. 0 maior poder explicativo das variáveis locais sugere um caráter auto-organizado dos Trending Topics, apontando caminhos para pesquisas futuras com base na epistemologia perspectivista. Tais resultados também podem ajudar a delinear métodos de ranqueamento e segmentação de usuários.

\section{Palavras-chave}

Rede social. Twitter. Perspectivismo. Formador de opinião. Software.

Rodrigo Travitzki | rodrigo@rizomas.net Doutorando em Educação na Universidade de São Paulo (USP).

Marco Toledo Bastos I opus@usp.br Pós-doutorando Fapesp na Universidade de São Paulo (USP).

\section{Rafael Luís Galdini Raimundo}

rafael.raimundo@gmail.com

Doutorando em Ecologia na Universidade de São Paulo (USP).

\section{Introdução}

\subsection{Perspectivismo nas redes sociais}

As mídias digitais permitem a convergência de outras mídias. A internet, um espaço global que cresceu com este princípio, tornou-se a maior fonte de informação disponível a uma pessoa. Neste espaço, surgiram condições tecnológicas para o florescimento de um novo tipo de temporalidade social, que vem se constituindo em torno do "tempo real" (LÉVY, 1993, p. 117).

Recentemente, a rapidez na criação e recepção de informações sofreu novo salto com o surgimento das redes sociais, que são sub-redes dentro da rede mundial caracterizadas por dinâmicas particulares. No caso das plataformas de microblog, como 0 Twitter, ${ }^{1}$ os usuários podem contribuir com custo zero e esforço mínimo (BAKSHY et al., 2011), gerando notícias, conceitos e outras ideias que podem adquirir repercussão ampla e rapidamente. Além disso, há evidências de que o simples número de menções no Twitter seja capaz de refletir 0 
resultado de uma eleição (TUMASJAN et al., 2011), o que abre novas possibilidades metodológicas para pesquisas de opinião que fundamentam estratégias de comunicação social, marketing, ou mesmo decisões de Estado.

Contudo, em meio a tanta informação, muito acaba se tornando ruído, tanto para os usuários quanto para os pesquisadores. Como filtrar o ruído e maximizar a informação? Para cada usuário, a rede social aparece e funciona de forma diferente, ou seja, existe uma espécie de "filtro personalizado" que permite a inteligibilidade e operacionalidade das redes sociais. Cada um vê a rede na sua própria perspectiva. Mas e para os pesquisadores, há algo parecido em termos de metodologia?

0 perspectivismo não é uma invenção da modernidade, ele pode ser encontrado, por exemplo, em antigas culturas ameríndias (VIVEIROS DE CASTRO, 1998). Segundo McGuire (1989, p. 217), a epistemologia perspectivista se fundamenta na ideia de que qualquer conhecimento é uma representação válida apenas em contextos limitados, e, portanto múltiplas teorias são necessárias. Mais ainda, o perspectivismo enriquece o empirismo, não limitando-o ao teste de hipóteses:

[...] a confrontação empírica é utilizada com mais eficácia, não para testar se certa hipótese é verdadeira, mas para descobrir seu significado [...] A confrontação empirica é melhor utilizada como uma continuação a posteriori do processo de descoberta que se inicia na análise conceitual a priori.
Numa abordagem perspectivista, os conceitos de verdade e objetividade não operam como no positivismo lógico (ANDERSON, 1998), não se trata simplesmente de testar se uma afirmação é verdadeira ou falsa, mas de buscar conhecer melhor o(s) significado(s) dessa afirmação.

A internet, estando em constante processo de auto-organização, talvez seja um terreno mais fértil para abordagens perspectivistas do que essencialistas (GRANIC; LAMEY, 2000).

Neste trabalho investigamos tal hipótese, apresentando os primeiros resultados de uma metodologia para análise dos usuários no Twitter, cujos princípios podem ser extrapolados para outras redes sociais. Foram comparadas, dentre outras, variáveis mais "essencialistas" (globais, a priori) e variáveis mais "perspectivistas" (locais, a posteriori).

\subsection{Informação no Twitter}

Que características ou comportamentos das pessoas estão mais associados à sua capacidade de disseminar informação? Nas redes sociais, há pelo menos dois tipos de causa, não excludentes, para tal eficácia comunicativa, que poderíamos chamar de "fator topológico" (relacionado a métricas mais complexas sobre a posição do usuário na rede) e "fator celebridade" (relacionado a métricas mais simples, como o número de seguidores ou de menções, por 
exemplo) (BOYD; GOLDER; LOTAN, 2010).

Pode-se classificar os usuários em três tipos: os produtores, os compartilhadores e os leitores de informação (MAZZOCATO, 2011). Neste estudo investigamos principalmente o primeiro tipo, potencializado pelo segundo.

0 Twitter vem se consolidando como base de pesquisas quantitativas sobre comunicação (WU et al., 2011). 0 principal mecanismo de disseminação de informação nesta rede social é 0 retuíte, e sua dinâmica já começa a ser estudada (SUH et al., 2010). A maior parte dos estudos se fundamenta na análise de (virtuamente) todos os tuítes em certo período de tempo (KWAK et al., 2010; ROMER0; MEEDER; KLEINBERG, 2011), o que permitiria a comparabilidade dos tuítes, assim como a análise do impacto mundial de certo evento.

Por outro lado, este método requer computadores com alta capacidade de processamento, além de acessos "especiais" aos dados do Twitter. ${ }^{2}$ Além disso, o foco nas características globais pode ocultar padrões locais, referentes a cada tema. Sendo assim, neste trabalho utilizamos uma metodologia diferente, coletando e analisando independentemente cada Trending Topic. Buscamos, com isso, a identificação de fatores locais relacionados à comunicação nas redes sociais, que possam fundamentar metodologias para ranqueamento e segmentação de usuários, para pesquisas de opinião espontânea a baixo custo, dentre outras.

Uma forma comum de investigar os mecanismos de disseminação da informação se fundamenta na identificação dos diferentes graus de influência das pessoas em uma rede. Há, contudo, diferentes variáveis relacionadas ao grau de influência, e nos últimos anos diversos trabalhos têm mapeado estas relações (BAKSHY et al., 2011; CHA et al., 2010; WENG et al., 2010; WU et al., 2011; YE; WU, 2010). Segundo estes autores, os principais indicadores de influência dos usuários no Twitter seriam:

a) número de seguidores, ou indegree influence (*);

b) número de retuítes $(*)$;

c) distribuição de cascatas de tuítes;

d) número de menções;

e) número de respostas recebidas (*) ou de respostas recebidas por usuários únicos; ${ }^{3}$ f) ranking do site (page rank).

Com o objetivo de compreender melhor a disseminação da informação nas redes sociais, investigamos aqui os fatores relacionados ao número de retuítes de cada usuário (b). Há evidências de que esta variável esteja entre as mais estáveis como indicador da influência dos usuários (YE; WU, 2010). 


\section{Metodologia}

\subsection{Plataforma T}

Para coletar (virtualmente) ${ }^{4}$ todos os tuítes que mencionam uma hashtag durante certo período, utilizamos o software YourTwapperKeeper $0.55,{ }^{5}$ licenciado em GNU General Public

License Ver. 2. Contudo, para obter informações detalhadas a respeito dos usuários que escreveram, receberam ou foram mencionados em cada tuíte, criamos alguns scripts em PHP e MySQL. A análise de dados, por sua vez, foi realizada em R, através de scripts articulados aos primeiros. Chamamos este sistema de plataforma T, representada na Figura 1.

0s scripts PHP tem o objetivo de coletar os dados ininterruptamente da internet, armazenando-os em tabelas MySQL. Estas são importadas para $0 \mathrm{R}$, onde podem ser analisadas de diversas maneiras, algumas das quais descrevemos a seguir. Observase a diversidade de APIs e articulação entre diferentes linguagens e ambientes de programação. Muitas informações pertinentes já podem ser obtidas com a análise automatizada (1). Se necessário, alguns scripts semi-prontos ajudam a realizar diferentes tipos de análise, customizada de acordo com 0 interesse (2).

Nossa amostra é composta exclusivamente de hashtags que se tornaram Trending Topic em 2011, com mínimo de dois mil e máximo de 50 mil tuítes. Cada um desses conjuntos de tuítes que mencionam uma hashtag em certo período se constitui numa unidade de análise, e os diversos dados recolhidos a respeito destes tuítes serão

Figura 1 - A plataforma T - um conjunto de scripts capaz de automatizar a análise de temas no Twitter - integrada ao programa YourTwapperKeeper e a diferentes APIs do Twitter.

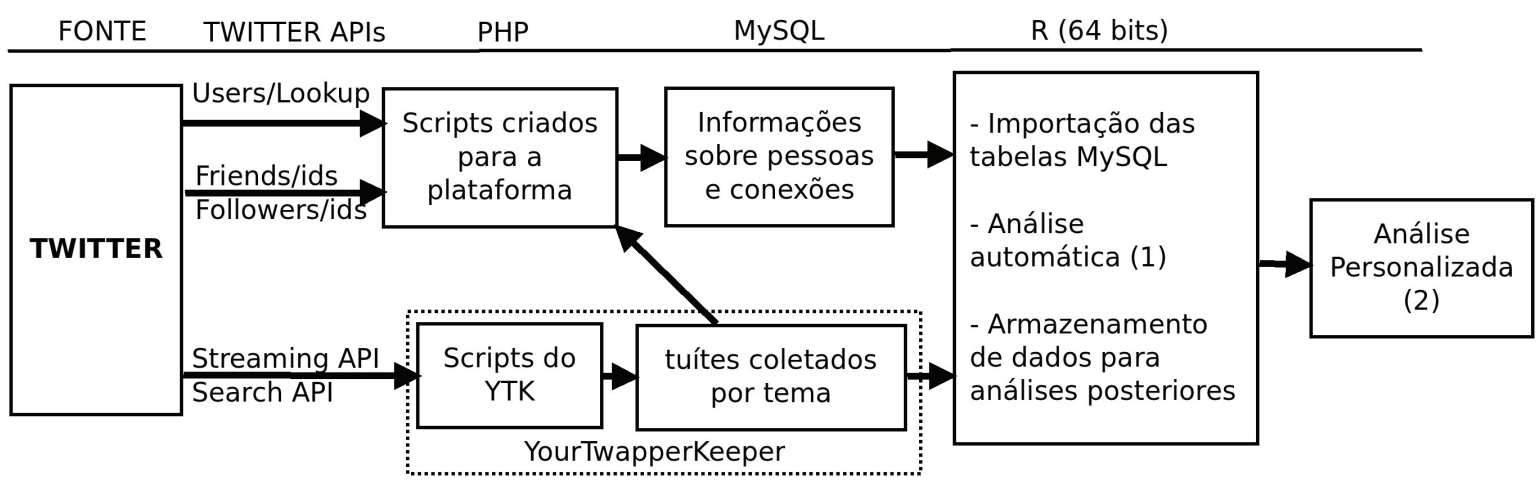

0 programa YourTwapperKeeper utiliza de forma complementar duas APIs para coletar todos os tuítes contendo certo termo, mas sobrecargas nos servidores do Twitter, dentre outros problemas técnicos, podem fazer com que uma ou outra mensagem deixe de ser coletada. 
chamados aqui de hashdata. 0 Quadro 1 mostra os 32 Trending Topics analisados neste trabalho. Construímos três redes diferentes para cada um deles: uma de retuítes (RT), uma de respostas (RP), e outra de amigos e seguidores locais dentro do hashdata - (LF), todas com conexões direcionadas no sentido da disseminação de informação. Foram contados os edges indegree e outdegree de cada usuário em cada rede e calculadas algumas métricas simples. Esta técnica permitiu trabalhar com variáveis locais (a posteriori) de acesso relativamente difícil, já que não estão diretamente acessíveis pelas APIs.

Além disso, coletamos diversas informações sobre os usuários (que escreveram os tuítes da amostra ou estão nela em retuítes ou respostas). Aqueles sem perfil público foram retirados da análise, tanto por questões técnicas quanto éticas. De cada usuário, foram obtidas as seguintes variáveis numéricas:

1) início da conta: tempo desde data de criação da conta no Twitter;

2) seguidores (global): número de seguidores no Twitter;

3) amigos (global): número de amigos no Twitter ;

4) status (global): número total de mensagens escritas no Twitter;

5) status (local): número de mensagens no hashdata;
6) seguidores (local) - LF out: número de seguidores no hashdata;

7) amigos (local) - LF in: número de amigos no hashdata;

8) retuítes - RT out: número de vezes que foi retuitado no hashdata;

9) retuítes enviados - RT in: número de vezes que retuíteou no hashdata;

10) respostas enviadas - RP out: número de mensagens pessoais enviadas no hashdata; 11) respostas recebidas - RP in: número de mensagens pessoais recebidas no hashdata. ${ }^{6}$

As variáveis locais não foram encontradas na literatura, que costuma se restringir às informações recebidas diretamente das APIs (como número global de seguidores, obtido pela API users/lookup). Talvez esta predileção por informações da API se deva às múltiplas dificuldades metodológicas que tivemos para elaborar os scripts da plataforma T, ou quem sabe porque este tipo de informação só seja válido dentro de seu próprio contexto, limitando seu uso como modelo preditivo.

É importante salientar ao menos duas peculiaridades do nosso método: 1) optamos por focalizar em características dos usuários relacionadas à estrutura geral do Twitter - as conexões, os retuítes e respostas (que correspondem às três redes construídas) -

As variáveis sublinhadas correspondem às marcadas $\left(^{\star}\right)$ na introdução. As variáveis de 1 a 4 foram obtidas diretamente das APIs do Twitter, enquanto as restantes foram calculadas com scripts específicos escritos para a plataforma T. Por fim, as variáveis de 6 a 11 correspondem a métricas simples (número de conexões - edges indegree e outdegree - de cada vértice, sendo os vértices os usuários) das três redes supramencionadas. 
mas específicas de um certo tema, dado que mencionam uma mesma hashtag. Com isto, foi possível chegar à segunda peculiaridade: 2) obtivemos algumas medidas qualitativamente semelhantes, porém em dois níveis diferentes - ou seja, no Twitter como um todo ou apenas na amostra colhida sobre um tópico específico (que chamamos de variáveis globais e locais, ou variáveis a priori e a posteriori, respectivamente). São três variáveis deste tipo, totalizando seis entre as onze mensuradas de cada usuário: o número de mensagens (statuses), o número de amigos e o número de seguidores (friends efollowers).

\subsection{Influência dos usuários}

Realizamos análise da influência dos usuários em termos do número de retuítes. Isso foi feito de forma independente em cada tema, para depois serem comparamos os valores obtidos nas 32 amostras. Avaliamos a importância das diversas características do usuário na probabilidade dele ser retuitado, vista como um indicador de sua influência na rede. $\mathrm{Ou}$ seja, trata-se de um recorte particular da pergunta mais geral: em que condições a informação tende a ser mais disseminada?

Nesta análise da influência dos usuários, iniciamos calculando as correlações (Pearson, $p<0,05)$ entre as 11 variáveis coletadas sobre cada usuário em cada hashdata. A partir destes resultados, escolhemos três variáveis potencialmente explicadoras do número de retuítes e ajustamos alguns modelos de regressão. Verificamos então as correlações (Pearson, $p<0,01$ ) destas três variáveis entre si, no intuito de respeitar os pressupostos da regressão multivariada, e optamos por ajustar apenas um modelo deste tipo. Foram quatro modelos diferentes em nossa análise de regressão, todos tendo o número de retuítes como variável resposta: três deles unidimensionais, correspondendo aos três indicadores selecionados, e um multivariado, integrando status (local) e seguidores (local) como variáveis explicativas (Figura 6). Foram ajustados, portanto, 128 modelos, quatro para cada um dos 32 hashdatas. De cada modelo, por sua vez, calculamos três valores - 0 AIC, 0 p e 0 coeficiente de determinação ajustado totalizando 384 medidas para comparação dos modelos.

Para comparação das variáveis locais e globais, utilizamos 0 teste $\mathrm{T}$ de Student com algumas das correlações supramencionadas. Antes disso, substituímos os dados faltantes por zeros, partindo do princípio de que quando $0 \mathrm{R}$ não conseguiu calcular a correlação, ela pode ser considerada nula.

\subsection{Segmentação}

Uma última técnica que merece ser mencionada é a que fundamenta a construção do dendrograma (Figura 2) ou, de maneira mais geral, a segmentação de usuários e temas, dependendo 
do que se deseja, a partir do mesmo conjunto de dados. É um procedimento razoavelmente simples de tabulação e agrupamento, fundamentado na sobreposição de usuários, ou seja, a ocorrência de certos usuários em mais de uma hashtag do conjunto analisado.

0 primeiro passo desta técnica consiste em coletar um certo conjunto de hashtags (ou palavras ou expressões), de acordo com objetivos definidos. Depois, para cada uma delas, identifica-se os usuários (utilizando expressões regulares ou informações das APIs, por exemplo), armazenando-os na coluna A de uma tabela. A coluna B, por sua vez, deve ser completamente preenchida com a mesma palavra, que correspondente à hashtag. Feito isso para todas as hashtags do conjunto, somase as tabelas verticalmente, resultando numa única tabela de duas colunas. A partir desta tabela geral é possível comparar tanto usuários quanto hashtags.

Em primeiro lugar, pode-se ranquear os usuários simplesmente tabulando a coluna A. Esta pode ser, inclusive, mais uma variável local a ser incluída em modelos da influência do usuário. Para segmentação das hashtags, contudo, são necessários mais procedimentos. Partindo dos mesmos dados, tabula-se os usuários por hashtag, criando uma matriz composta de zeros e uns, de U linhas por H colunas (sendo U o número de usuários únicos e $\mathrm{H}$ o número de hashtags do conjunto previamente selecionado). Na teoria dos grafos, formalmente esta seria uma matriz de adjacência de um bigraph, um grafo bipartido (HARARY, 1969). A partir desta matriz é construída uma matriz de distâncias, que por sua vez permite a segmentação. Para 0 dendrograma da Figura 2, utilizamos respectivamente os métodos aka asymmetric binary e ward, porém outros métodos estão disponíveis tanto para 0 cálculo das distâncias (BORG; GROENEN, 1997) quanto para segmentação (HARTIGAN, 1975). Caberia, em futuros estudos, uma comparação de tais métodos. Por fim, a partir desta segmentação de hashtags pode-se também elaborar uma segmentação dos usuários presentes nelas. Haveria, com isso, três possíveis utilidades para os dados presentes na referida tabela geral: ranquear usuários, segmentar temas e, consequentemente, segmentar usuários.

\section{Resultados}

Analisamos 595.240 mensagens - dos quais 217.344 eram retuítes e 51.817 respostas

- distribuídas em 32 Trendings Topics com tamanho variável entre dois mil e 50 mil tuítes cada. A maioria em português, com exceção de três (\#notenemosmiedo, \#DemiYouAreBeautiful e \#misstwitter). Apesar disso, os hashdata apresentaram cerca de dez idiomas cada um, refletindo certa internacionalidade do twitter. A Quadro 1 mostra os 32 temas e suas variáveis básicas. 
Quadro 1 - Resumo dos hashdatas. Cada hashdata corresponde a uma série de variáveis obtidas a partir de um conjunto de tuítes sobre certo Trending Topic

\begin{tabular}{|c|c|c|c|c|c|c|}
\hline Hashtag & $\begin{array}{c}\text { Início da } \\
\text { coleta }\end{array}$ & $\begin{array}{c}\text { Duração } \\
\text { (dias) }\end{array}$ & $\begin{array}{l}\text { Número de } \\
\text { usuários }\end{array}$ & Tuítes & Retuítes & $\begin{array}{c}\text { Respostas } \\
\text { pessoais }\end{array}$ \\
\hline \#1bomprofessormeensinou & $12 / 04 / 2011$ & 78 & 16855 & 28697 & 17498 & 233 \\
\hline \#abaixodecreto & $22 / 02 / 2011$ & 93 & 11372 & 44813 & 17274 & 13466 \\
\hline \#adoteumanimalabandonado & 02/07/2011 & 62 & 1879 & 3131 & 1448 & 107 \\
\hline \#amorodeio & $23 / 08 / 2011$ & 8 & 8079 & 16725 & 6325 & 1631 \\
\hline \#battisti & 08/06/2011 & 27 & 3309 & 6434 & 2658 & 365 \\
\hline \#DemiYouAreBeautiful & $29 / 08 / 2011$ & 5 & 7736 & 11971 & 3915 & 1283 \\
\hline \#diadoadvogado & $11 / 08 / 2011$ & 17 & 10513 & 12689 & 4595 & 587 \\
\hline \#diadofrevo & 09/02/2011 & 35 & 2227 & 3601 & 1198 & 367 \\
\hline \#diadoreporter & $16 / 02 / 2011$ & 32 & 4927 & 6596 & 2070 & 1047 \\
\hline \#diamundialsemtabaco & $31 / 05 / 2011$ & 34 & 9223 & 14320 & 3994 & 600 \\
\hline \#escolhiesperaremdeus & 05/07/2011 & 49 & 15048 & 27274 & 13068 & 653 \\
\hline \#estudarvaleapena & $11 / 08 / 2011$ & 29 & 19798 & 26638 & 9742 & 791 \\
\hline \#fichalimpa & $23 / 03 / 2011$ & 69 & 17034 & 32255 & 11659 & 1597 \\
\hline \#forabolsonaro & $31 / 03 / 2011$ & 76 & 18030 & 30526 & 11038 & 1819 \\
\hline \#frasesqueeikenuncadiz & $12 / 02 / 2011$ & 38 & 1821 & 3929 & 1034 & 219 \\
\hline \#menoscormaisrock & 09/05/2011 & 119 & 5113 & 15472 & 4354 & 2649 \\
\hline \#misstwitter & $27 / 06 / 2011$ & 54 & 12951 & 35774 & 8549 & 12148 \\
\hline \#naofoiacidente & 01/03/2011 & 86 & 5892 & 15124 & 8133 & 710 \\
\hline \#notenemosmiedo & $19 / 05 / 2011$ & 26 & 14901 & 33908 & 19179 & 1586 \\
\hline \#odeiorodeio & $23 / 08 / 2011$ & 6 & 13134 & 26988 & 12400 & 1921 \\
\hline \#otwittermeensinou & 09/01/2011 & 74 & 924 & 2199 & 1243 & 15 \\
\hline \#pedofilianao & $27 / 06 / 2011$ & 54 & 3908 & 6308 & 2939 & 665 \\
\hline \#qndomertiolateardia & $27 / 05 / 2011$ & 27 & 8455 & 15839 & 4772 & 399 \\
\hline \#realengo & 08/04/2011 & 126 & 19470 & 33105 & 11602 & 2247 \\
\hline \#seeufosserico & 09/07/2011 & 50 & 16418 & 24440 & 5874 & 381 \\
\hline \#sosnatal & $15 / 06 / 2011$ & 16 & 2495 & 6318 & 3136 & 459 \\
\hline \#trabalhoescravo & $17 / 08 / 2011$ & 19 & 3496 & 4315 & 2020 & 154 \\
\hline \#tuíteeumsonho & $15 / 06 / 2011$ & 5 & 19418 & 26574 & 6165 & 591 \\
\hline \#tuíteumlivro & $27 / 05 / 2011$ & 5 & 28411 & 43960 & 6311 & 577 \\
\hline \#tuíteumnomeestranho & $18 / 02 / 2011$ & 30 & 5102 & 8095 & 1009 & 393 \\
\hline \#tuíteumproverbio & 09/05/2011 & 17 & 6000 & 10474 & 4146 & 213 \\
\hline \#uniaohomoafetiva & 06/05/2011 & 22 & 11593 & 16748 & 7996 & 1944 \\
\hline
\end{tabular}


Observa-se que o número de respostas é sistematicamente menor que 0 de retuítes, confirmando o papel destes, como principal mecanismo do Twitter (SUH et al., 2010). A proporção de retuítes nos 32 Trending Topics apresentou uma distribuição normal ( $\mathrm{X}=0,38$, $\mathrm{SD}=0,12)$, com mínimo de $12,5 \%$ e máximo de $61,0 \%$. Esta proporção parece maior do que em estudo anterior (KWAK et al., 2010).

\subsection{Segmentação de usuários e temas}

Para analisar os fatores que influenciam a disseminação da informação nas redes sociais, focalizamos a investigação nas características dos usuários (que tuitaram, receberam respostas ou foram retuitados) de cada hashdata. Alguns usuários foram encontrados em mais de um Trending Topic.

Tabela 1 - Recorrência dos usuários ao longo das 32 hashtags

\begin{tabular}{|c|c|c|}
\hline $\begin{array}{c}\text { TTs em que } \\
\text { foi citado }\end{array}$ & $\begin{array}{c}\text { Número } \\
\text { de Usuários }\end{array}$ & N.U. (\%) \\
\hline 1 & 218047 & 83,3 \\
\hline 2 & 31253 & 11,94 \\
\hline 3 & 8095 & 3,09 \\
\hline 4 & 2642 & 1,01 \\
\hline 5 & 949 & 0,36 \\
\hline 6 a 25 & 771 & 0,29 \\
\hline
\end{tabular}

Foram analisados 261.757 usuários, dos quais 16,7 \% foram encontrados em mais de uma das 32 hashdatas, o que sugere certa sobreposição entre o público envolvido nos temas aqui selecionados. É possível utilizar estas informações como forma de agrupamento de hashtags ou de usuários a partir do mesmo conjunto de dados, escolhendo cuidadosamente o conjunto de hashtags/ palavras a ser coletado continuamente pela plataforma T. Esta técnica pode ser eficaz para pesquisas de opinião e de segmentação com base no Twitter.

Podemos partir do principio de que a sobreposição de usuários seria mais comum em hashtags semelhantes em algum sentido. Apenas para ilustrar as possibilidades, a Figura 2 mostra um dendrograma agrupando os temas em função da coocorrência de usuários entre eles.

É interessante observar que duas hashtags com conteúdo oposto mostram-se bastante próximas em termos de recorrência de usuários (\# amorodeio e \# odeiorodeio). Também notase certa tendência de agrupamento de temas "políticos" (\#forabolsonaro, \#fichalimpa, \#battisti, \# abaixodecreto, \# naofoiacidente, \# realengo). Estes são, contudo, resultados preliminares, utilizando um entre diversos métodos de agrupamento. Novos estudos podem ser feitos para investigar a eficácia dos diferentes métodos dependendo da finalidade (segmentação de temas ou de usuários, ou ranqueamento de usuários).

Os resultados e a própria eficácia deste método dependem, dentre outras, da escolha do conjunto de temas, o que reflete seu caráter perspectivista. 
Figura 2 - Dendrograma ilustrando possibilidade de agrupamento de hashtags em função da sobreposição de usuários.

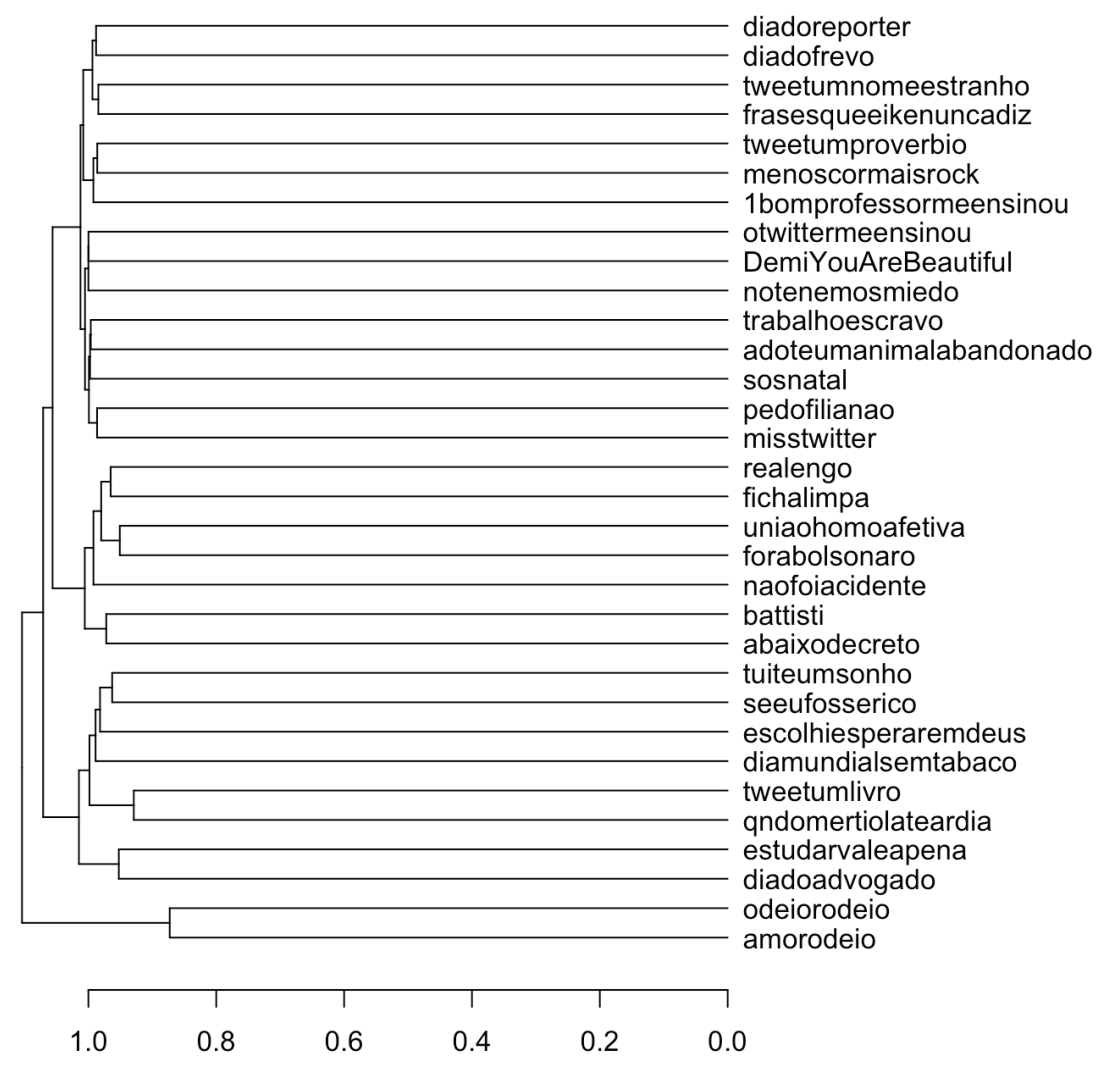

\subsection{A disseminação da informaç̧ão}

Das 11 variáveis obtidas de cada usuário, uma foi o número de retuítes no hashdata, considerado aqui como o principal indicador de influência do usuário - sua capacidade de disseminar informação. Nesse sentido, consideramos as outras dez variáveis como explanatórias em potencial. Vejamos uma amostra dos dados brutos para ter uma ideia mais clara do que foi feito. A Quadro 2 mostra algumas das variáveis obtidas de cada usuário no Trending Topic "\#diadofrevo".
Observa-se que o número de retuítes (RT) parece ser explicado por diferentes variáveis dependendo do usuário.0 mais retuitado (@ marceloadnet0) é um famoso humorista de TV, e poderia ser detectado com qualquer um dos indicadores (seguidores globais, locais, ou respostas recebidas).0 segundo usuário (@ carnavalrecife), contudo, está mais intimamente relacionado à hashtag (dado que Recife é considerada a capital do frevo, uma dança típica brasileira), e seria melhor detectado pelos seguidores locais do que pelos globais. 0 terceiro, por sua vez, embora tenha poucos 
Quadro 2 - Exemplo dos dados brutos colhidos sobre cada usuário em cada hashdata

\begin{tabular}{|c|c|c|c|c|c|c|}
\hline Nome do usuário & RT & $\begin{array}{c}\text { Status } \\
\text { (global) }\end{array}$ & $\begin{array}{c}\text { Seguidores } \\
\text { (global) }\end{array}$ & $\begin{array}{c}\text { Status } \\
\text { (local) }\end{array}$ & $\begin{array}{c}\text { Seguidores } \\
\text { (local) }\end{array}$ & Respostas recebidas \\
\hline @marceloadnet0 & 261 & 1360 & 657218 & 1 & 330 & 8 \\
\hline @carnavalrecife & 75 & 2661 & 3608 & 19 & 121 & 9 \\
\hline @giiorgio_ & 44 & 3142 & 389 & 1 & 0 & 11 \\
\hline @lucas22monart & 24 & 3685 & 217 & 42 & 2 & 2 \\
\hline @vanessa_violeta & 18 & 566 & 402 & 1 & 12 & 0 \\
\hline @c_amaraloficial & 14 & 820 & 261 & 36 & 0 & 6 \\
\hline @frevodepressivo & 13 & 106 & 769 & 2 & 56 & 1 \\
\hline @edizinhah & 9 & 16756 & 183 & 33 & 5 & 0 \\
\hline
\end{tabular}

seguidores, recebeu muitas respostas. E 0

quarto usuário (@lucas22monart),por fim,não seria detectado com nenhum dos indicadores de influência. No entanto, observamos que ele tuitou muitas mensagens com aquela hashtag. Este usuário pode ser considerado um exemplo do esforço pessoal gerando certa quebra no padrão espontâneo de disseminação da informação (fortemente influenciado pelo "fator celebridade"), o que poderia estar relacionado a um papel político de plataformas como o Twitter ou redes sociais em geral.

Para verificar as hipóteses ilustradas acima, inicialmente calculamos as correlações entre todas as variáveis em cada hashdata. Separamos, então, os resultados referentes às correlações entre as dez variáveis potencialmente explicativas e 0 número de retuítes. Feito isto, contamos o número de correlações significativas $(p<0,05)$ nos 32 hashdatas e calculamos suas médias (Figura 3).
Nota-se que as correlações são baixas e muitas vezes raras, mas há algumas variáveis explicativas em potencial. Em que medida estes resultados confirmam resultados de estudos anteriores? Das 11 variáveis que obtivemos para cada usuário, três são consideradas na literatura especializada como potenciais indicadores de influência. Destas três, uma é o próprio número de retuítes, restando, portanto, outras duas a serem testadas como possíveis variáveis explicativas, ou indicadores deste. Na Figura 3, o número de respostas é a variável mais correlacionada ao número de retuítes, confirmando o que já se supunha. Outro resultado que confirma estudos anteriores (SUH et al., 2010) é a irrelevância do tempo de existência da conta no Twitter para o grau de influência do usuário.

\subsection{Variáveis "globais" e "locais"}

Na segunda variável potencialmente indicadora do retuíte (o número de seguidores) as correlações já não parecem tão consistentes, ao menos quando 
Figura 3 - Correlações entre as possíveis variáveis explicativas e o número de retuítes de cada usuário. Observa-se 0 número de correlações válidas (A) e a média destas (B).

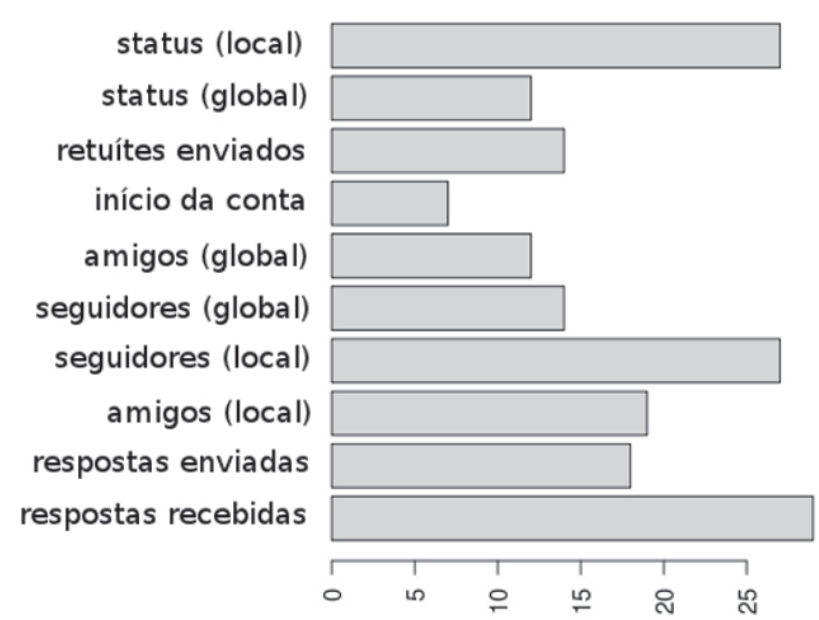

(A) Número de correlações significativas $(p<0,05)$

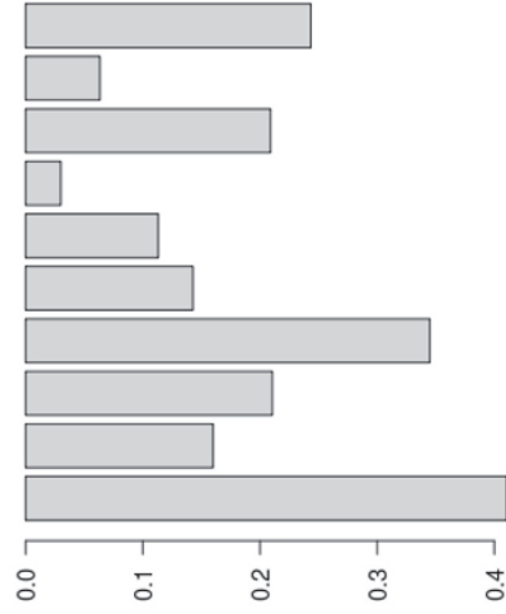

(B) Média das correlações significativas observamos sua "versão global". Contudo, quando observamos o número de seguidores locais, ou seja, dentro de um tema específico, parece haver correlações mais sólidas. Isso acontece nas três variáveis que possuem versões "locais" e "globais" (seguidores, amigos e status - que são as mensagens, os tuítes).

\section{A "versão global" das variáveis corresponde aos} três números que se vê quando se clica em alguém dentro do próprio twitter. Como estes dados podem ser obtidos antes de se arquivar tuítes específicos de uma hashtag, foram aqui considerados variáveis a priori. A "versão local" da variável foi calculada com scripts específicos da plataforma T, a partir de um certo hashdata, ou seja, só pode ser obtida a posteriori. Pois bem, em que medida as aparentes diferenças entre esses três pares de variáveis são estatisticamente consistentes?
Comparando as versões global e local de cada par, 0 teste $\mathrm{T}$ de Student revela diferença em todos, mais significativas entre número de status $\left(p=2 e^{-}\right.$ $5)$, seguidores ( $p=2 e-5)$ e um pouco menor entre amigos $(p=0,008) .0 u$ seja, as variáveis locais (a posteriori) apresentam, em média, maior correlação com 0 retuíte do que as variáveis globais (a priori), quando comparamos variáveis do mesmo tipo.

Isso sugere que o grau de influência do retuíte em um assunto está mais relacionado à dinâmica interna do próprio assunto do que a uma estrutura geral anterior ou exterior a ela. Em outras palavras, a comparação das variáveis globais e locais (Figura 3) sugere que a dinâmica do retuíte é mais influenciada por fatores criados durante o processo do que por aqueles dados a prioricaracterística presente, por exemplo, em processos 
auto-organizados (DEBRUN, 1996) e propriedades emergentes (KIM, 1993, p. 346). A auto-organização seria um aumento espontâneo de ordem gerado nos pontos de instabilidade, ou pontos de bifurcação, e está intimamente relacionada aos fenômenos vivos e suas manifestações (CAPRA, 2002). Embora apresente certa imprevisibilidade a priori, relacionada às bifurcações, a auto-organização pode ser analisada a posteriori, utilizado-se variáveis criadas durante o próprio processo.

Este é um possível pano de fundo teórico para se investigar as redes sociais como espaço heterogêneo, de onde emergem continuamente fenômenos locais que podem ser melhor compreendidos a partir de seus próprios parâmetros - ou seja, em uma abordagem perspectivista (MCGUIRE, 1989). Podemos dizer, portanto, que esses resultados confirmam a hipótese de que o perspectivismo pode ser mais frutífero do que o essencialismo na análise da internet (GRANIC; LAMEY, 2000) - especialmente das redes sociais.

Enfim, todos esses elementos confirmam a ideia de que 0 Twitter é um ambiente da velocidade, da criação contínua de sub-redes dentro da rede, mais explicáveis por suas características temporárias do que pelas propriedades isoladas dos elementos que as compõem, ou variáveis já conhecidas de antemão. Tal concepção pode ajudar a compreender a dificuldade de se ajustar modelos capazes de prever a dinâmica da informação nas redes sociais. Neste sentido, a investigação aqui desenvolvida talvez tenha mais a contribuir com a análise $a$ posteriori de temas do que com o desenvolvimento de algoritmos para estimar padrões futuros. 0 que poderia ser útil na elaboração de pesquisas de opinião e mercado com base em informações disponibilizadas espontaneamente pelas pessoas um novo ramo que surgiu com os blogs, microblogs e redes sociais.

\subsection{Detectando formadores de opinião}

A partir da análise de correlações, selecionamos algumas variáveis para análise de regressão. Observando as médias da Figura 3, duas variáveis se destacam: 1) o número local de seguidores, que se mostrou mais significativo do que 0 global - que parece ser importante (YE; WU, 2010), mas menos do que se supõe (CHA et al., 2010); e 2) o número de respostas recebidas - fator considerado importante tanto no Twitter (BOYD; GOLDER; LOTAN, 2010) como em outras redes sociais (RECUERO, 2005). Contudo, se observamos também o número de correlações significativas, um terceiro fator potencialmente significativo aparece: 3) o número de status na amostra. Esta terceira variável, ao contrário das duas primeiras, não foi encontrada na literatura como um fator importante para o grau de influência dos usuários.

Identificados três fatores, começamos verificando as correlações entre eles. As correlações médias (Pearson, $p<0,05$ ) entre os três indicadores potenciais ao longo das 
32 hashdatas foram: 0,27 entre respostas e seguidores; 0,22 entre respostas e status; 0,13 entre status e seguidores. A média mais alta encontrada nas correlações entre respostas recebidas e seguidores locais confirma a ideia de que eles podem ser indicadores de uma mesma propriedade, possivelmente relacionados ao "fator celebridade". 0 número de status (local), por sua vez, é um pouco menos correlacionado com os outros, sugerindo que ele pode ser uma fonte adicional de informações (pouco redundante), especialmente se articulado ao número de seguidores (local). A partir dessas observações, optamos por ajustar quatro tipos de modelos para cada hashdata, três dos quais univariados (tendo os três indicadores como variáveis independentes e o retuíte como variável dependente) e um multivariado.

Ao se comparar os valores de $\mathrm{p}$ dos modelos, constata-se que o modelo multivariado foi inválido ( $p>0,001)$ em apenas duas das 32 hashtags, enquanto o modelo por respostas o foi em três. Os outros dois modelos foram inválidos cinco vezes cada um. São resultados pouco diferentes, mas mostram a significância estatística dos dois primeiros modelos.

Comparando os AICs apenas dos modelos unidimensionais, em 16 hashdatas o número de respostas recebidas explicava melhor o retuíte que os outros dois. Em 14 hashdatas o número de seguidores locais explicou melhor o retuíte, restando ao número de status locais apenas dois dos 32 TTs. A comparação dos coeficientes de determinação apresentou o mesmo resultado. Por fim, quando incluímos o modelo multivariado na comparação, ele é considerado 16 vezes o melhor, seguido do modelo por "respostas" (14 vezes) e por seguidores locais (2 vezes).

Por fim, a comparação dos coeficientes de determinação revela grande variação (Quadro 3), embora haja diferenças significativas ${ }^{7}$ entre respostas recebidas e status ( $p<0,001)$, mas principalmente, como esperado, entre o modelo multidimensional e 0 de respostas recebidas $(p<2 e-8)$, seguido pelo de status $(p<0,001)$ e menos pelo de seguidores $(p<0,01)$.

Quadro 3 - Coeficientes de determinação dos modelos para o retuíte

\begin{tabular}{|c|c|c|}
\hline Modelo & Média & $\begin{array}{r}\text { Desvio } \\
\text { Padrão }\end{array}$ \\
\hline Respostas recebidas & 0,21 & 0,24 \\
\hline Seguidores (local) & 0,14 & 0,18 \\
\hline Status (local) & 0,08 & 0,13 \\
\hline Seguidores + Status & 0.20 & 0.21 \\
\hline
\end{tabular}

\section{Conclusões}

Esse trabalho teve dois objetivos: 1) apresentar e testar a Plataforma T, desenvolvida especialmente para coleta e análise de dados do Twitter; 2) 
mapear os indicadores de influência dos usuários, comparando variáveis locais e globais como forma de avaliar abordagens perspectivistas e essencialistas.

Sobre o primeiro objetivo, a estrutura da plataforma T (integrando Twitter API, PHP/MySQL e R) se mostrou confiável e flexível, captando tuítes de forma seletiva e contínua, realizando análises automáticas e também customizadas. A obtenção de resultados esperados confirma a qualidade dos dados coletados e eficácia dos métodos.

Na análise da influência dos usuários, consideramos o número de retuítes em certo tema como principal indicador, a partir do qual mapeamos a importância de outros fatores. Das dez variáveis numéricas de cada usuário (em um tema específico), foram identificadas três mais correlacionadas ao número de retuítes, em ordem de importância:

1)RP: o número de respostas (mensagens pessoais) recebidas dentro do tema (no caso, uma hashtag); 2) SE: seguidores (local), o número de seguidores entre as pessoas que tuitaram a hashtag;

3) ST: status (local), o número de tuítes com a hashtag.

A análise de regressão das três variáveis revelou que em diferentes Trending Topics o retuíte pode ser melhor explicado por um ou outro fator, e raramente por nenhum deles. Não obstante esta variação, os dois melhores modelos entre os quatro testados foram significativos $(p<0,01)$ em mais de $90 \%$ das amostras. Confirmando os resultados da análise de correlação, a variável mais capaz de explicar 0 retuíte foi RP (21\%), seguido de SE, que explicou em média $17 \%$ da variação no número de retuítes. A terceira variável (ST) explicou, em média, apenas $7 \%$ do retuíte, mas sua baixa correlação com SE permitiu a criação do modelo multivariado, juntando conexões locais (SE) e esforço (ST). Este modelo explicou, em média, $20 \%$ da variação do retuíte, com verossimilhança (AIC) sutilmente melhor que o modelo unidimensional por RP. Tais resultados podem ajudar a delinear métodos de ranqueamento e segmentação de usuários.

Neste sentido, construímos um dendrograma representando diferentes níveis de agrupamento das hashtags, como resultado preliminar para ilustrar as potencialidades do método. Tal análise só foi possível porque cerca de 17\% dos usuários analisados ocorreram em mais de uma das 32 hashtags, embora elas não tenha sido coletadas como se fizessem parte de um mesmo assunto. Tal sobreposição permite 0 uso do método de segmentação de assuntos por coocorrência de usuários, ou o inverso, segmentação de usuários por coocorrência de hashtags. Ambos partindo dos mesmos dados, referentes a um conjunto de temas escolhidos pelo pesquisador - abrindo espaço para uma abordagem perspectivista.

Nas três variáveis "duplas" (o número de status, de amigos e seguidores) a versão local se mostrou mais correlacionada ao número de retuítes do que a versão global, sugerindo que a disseminação da informação tem uma dinâmica pouco determinada 
a priori, mas que pode ser analisada a posteriori, a partir de parâmetros criados durante o próprio processo. Neste sentido, confirmando a hipótese de Granic e Lamey (2000), o perspectivismo pode se constituir num importante pano de fundo teórico para análise da internet.

Estes resultados são importantes no delineamento de metodologias para mapear a disseminação da informação e a influência nas redes sociais, que por sua vez podem auxiliar nas investigações nesta área, assim como na qualificação das pesquisas de opinião pública com base na internet.

\section{Referências}

ANDERSON, R. Truth and Objectivity in

Perspectivism. Synthese, v. 115, n. 1, p. 1-32, 1 abr. 1998.

BAKSHY, E.; HOFMAN, J.; MASON, W.; WATTS,

D. Identifying "Influencers" on Twitter. In: ACM

INTERNATIONAL CONFERENCE ON WEB SEACH

AND DATA MINING (WSDM). 4., 2011. New York.

Proceedings... New York: ACM, 2011.

BORG, I.; GROENEN, P. Modern Multidimensional

Scaling. Theory and Applications. New York: Springer, 1997.

BOYD, D.; GOLDER, S.; LOTAN, G. Tweet, tweet, retweet: Conversational aspects of retweeting on twitter. In: HAWAII INTERNATIONAL CONFERENCE

ON SYSTEM SCIENCES (HICSS-43), 43., 2010.

Kauai. Proceedings... Kauai: IEEE, 2010. Disponível em: <http://ieeexplore.ieee.org/xpls/abs_all.

jsp?arnumber $=5428313>$. Acesso em: 4 jan. 2012, 2010.

CAPRA, F. As conexões ocultas. São Paulo: Editora Cultrix, 2002.

CHA, M. et al. Measuring user influence in Twitter: The million follower fallacy. In: INTERNATIONAL AAAI
CONFERENCE ON WEBLOGS AND SOCIAL MEDIA,

4,, 2010. Washington. Proceedings... Washington: AAAI, 2010. Disponível em: < http://www.aaai.org/ocs/index. php/ICWSM/ICWSM10/paper/download/1538/1826>. Acesso em: 4 jan. 2012, 2010.

DEBRUN, M. A dinâmica da auto-organização primária. Auto-organização: estudos interdisciplinares, Centro de Lógica, Epistemologia e História da Ciência. Campinas: Unicamp, 1996. (Coleção CLE n. 18).

GRANIC, I.; LAMEY, A. V. The self-organization of the Internet and changing modes of thought. New Ideas in Psychology, v. 18, n. 1, p. 93-107, 2000.

HARARY, F. Determinants, permanents and bipartite graphs. Mathematics Magazine, v. 42, n. 3, 1969.

HARTIGAN, J. A. Clustering Algorithms. New York: Wiley, 1975.

KIM, J. Supervenience and mind: selected philosophical essays. New York: Cambridge University Press, 1993.

KWAK, H. et al. What is Twitter, a social network or a news media? In: INTERNATIONAL CONFERENCE ON WORLD WIDE WEB. 19., 2010. New York Proceedings... New York: ACM, 2010.

LÉVY, P. Tecnologias da inteligência: o futuro do pensamento na era da informática. Rio de Janeiro: Editora 34, 1993.

MAZZ0CATO, S. B. Os Papéis do Sujeito com Relação a Conteúdos Midiáticos no Tweetdeck: o produtor, 0 compartilhador eo leitor. E-compós, v. 14, n. 2, 2011.

MCGUIRE, W. J. A perspectivist approach to the strategic planning of programmatic scientific research. The psychology of science: Contributions to metascience. New York: Cambridge University Press, 1989. p. 214-245.

RECUER0, R. 0 capital social em redes sociais na Internet. Revista FAMECOS, n. 28, p. 88-106, 2005.

ROMERO, D. M.; MEEDER, B.; KLEINBERG, J. Differences in the Mechanics of Information Diffusion 
Across Topics: Idioms, Political hashtags, and Complex Contagion on Twitter. In: ACM INTERNATIONAL

WORLD WIDE WEB CONFERENCE, 20., 2011.

Hyderabad. Proceedings... Hyderabad: ACM, 2011.

SUH, B. et al. Want to be Retweeted? \{Large\} Scale Analytics on Factors Impacting Retweet in \{Twitter\} Network. In: INTERNATIONAL CONFERENCE

ON SOCIAL COMPUTING (SocialCom), 2., 2010.

Minneapolis. Proceedings... Minneapolis: IEEE, 2010.

TUMASJAN, A. et al. Election Forecasts With Twitter: How 140 Characters Reflect the Political Landscape. Social Science Computer Review, v. 29, n. 4, p. 402-418, 2011.

VIVEIROS DE CASTR0, E. Cosmological Deixis and Amerindian Perspectivism. The Journal of the Royal Anthropological Institute, v. 4, n. 3, p. 469-488, 1998. WENG, J. et al. Twitterrank: finding topic-sensitive influential twitterers. In: ACM INTERNATIONAL CONFERENCE ON WEB SEARCH AND DATA MINING, 3., 2010. New York. Proceedings... New York: ACM, 2010.

WU, S. et al. Who says what to whom on twitter. In: INTERNATIONAL CONFERENCE ON WORLD WIDE WEB, 20., 2011. New York. Proceedings... New York: ACM, 2011.

YE, S.; WU, S. Measuring message propagation and social influence on Twitter. com. Social Informatics: Lecture Notes in Computer Science, v. 6430, p. 216-231, 2010. 


\begin{tabular}{|c|c|}
\hline $\begin{array}{l}\text { Perspectivism as a } \\
\text { basis for social network } \\
\text { analysis: some results } \\
\text { from Twitter }\end{array}$ & $\begin{array}{l}\text { El perspectivismo como base } \\
\text { para métodos de análisis } \\
\text { de redes sociales: algunos } \\
\text { resultados con Twitter }\end{array}$ \\
\hline $\begin{array}{l}\text { Abstract } \\
\text { This article presents a methodology for analysis } \\
\text { of variables that influence the dissemination of } \\
\text { information on the social network Twitter. We } \\
\text { analyze data from } 595,240 \text { messages produced by } \\
261,757 \text { users and we found three local variables } \\
\text { related to the degree of replication of messages } \\
\text { (retweet): } 1 \text { ) the number of responses received } \\
\text { (which explained an average of } 21 \% \text { of the } \\
\text { retweets); } 2 \text { ) the number of followers in the sub- } \\
\text { network (17\%) and 3) the number of tweets with } \\
\text { the hashtag ( } 7 \% \text { ). Comparing a priori variables } \\
\text { (global) and posteriori ones (local), there was a } \\
\text { significant difference in the number of followers } \\
\text { of messages ( }<<0.001 \text { ) and also in the number of } \\
\text { friends (p }<0.01) \text {. The higher explanatory power } \\
\text { of the local variables suggests a self-organized } \\
\text { character of the Trending Topics, indicating } \\
\text { paths for future research based on perspectivist } \\
\text { epistemology. These results can also help to } \\
\text { delineate methods for ranking and segmentation } \\
\text { of users. }\end{array}$ & $\begin{array}{l}\text { Resumen } \\
\text { En este artículo presentamos una metodología } \\
\text { de análisis de variables que influencian la } \\
\text { diseminación de información en la red social } \\
\text { Twitter. Analizamos datos de } 595.240 \text { mensajes } \\
\text { producidas por } 261.757 \text { usuarios y encontramos } \\
\text { tres variables locales relacionadas a los niveles } \\
\text { de replicación de los mensajes: } 1 \text { ) el número } \\
\text { de respuestas recibidas (explicando } 21 \% \text { de } \\
\text { los mensajes replicados); 2) el número de } \\
\text { seguidores en la subred (17\%) y } 3 \text { ) el número de } \\
\text { mensajes con la hashtag. La comparación entre } \\
\text { las variables a priori (globales) y a posteriori } \\
\text { (locales) enseña una diferencia significativa } \\
\text { en el numero de seguidores, de mensajes } \\
\text { (p<0,001) y también en el número de amigos } \\
\text { (p<0,01). El poder explicativo más grande de } \\
\text { las variables locales sugiere una propiedad } \\
\text { de auto organización de los Trending Topics, } \\
\text { indicando caminos para investigaciones futuras } \\
\text { desde la epistemología del perspectivismo. Los } \\
\text { resultados aquí presentados también pueden } \\
\text { apoyar el diseño de métodos de clasificación y } \\
\text { segmentación de usuarios. } \\
\text { Palabras claves } \\
\text { Red social. Twitter. Perspectivismo. Formador de } \\
\text { opinión. Software. }\end{array}$ \\
\hline
\end{tabular}




\section{Expediente}

A revista E-Compós é a publicação científica em formato eletrônico da Associação Nacional dos Programas de Pós-Graduação em Comunicação (Compós). Lançada em 2004, tem como principal finalidade difundir a produção acadêmica de pesquisadores da área de Comunicação, inseridos em instituições do Brasil e do exterior.
E-COMPÓS I www.e-compos.org.br I E-ISSN 1808-2599

Revista da Associação Nacional dos Programas de Pós-Graduação em Comunicação.

E-compós, Brasília, v.15, n.3, set./dez. 2012.

A identificação das edições, a partir de 2008 passa a ser volume anual com três números.

\section{CONSELHO EDITORIAL}

Afonso Albuquerque, Universidade Federal Fluminense, Brasil Alberto Carlos Augusto Klein, Universidade Estadual de Londrina, Brasil Álvaro Larangeira, Universidade Tuiuti do Paraná, Brasil André Luiz Martins Lemos, Universidade Federal da Bahia, Brasil Ângela Freire Prysthon, Universidade Federal de Pernambuco, Brasil Angela Cristina Salgueiro Marques, Faculdade Cásper Líbero (São Paulo), Brasil Antonio Roberto Chiachiri Filho, Faculdade Cásper Líbero, Brasil Arthur Autran Franco de Sá Neto, Universidade Federal de São Carlos, Brasi Benjamim Picado, Universidade Federal Fluminense, Brasil César Geraldo Guimarães, Universidade Federal de Minas Gerais, Brasil Cristiane Freitas Gutfreind, Pontifícia Universidade Católica do Rio Grande do Sul, Brasil

Denilson Lopes, Universidade Federal do Rio de Janeiro, Brasil Eduardo Peñuela Cañizal, Universidade Paulista, Brasil

Eduardo Vicente, Universidade de São Paulo, Brasil

Eneus Trindade, Universidade de São Paulo, Brasil

Erick Felinto de Oliveira, Universidade do Estado do Rio de Janeiro, Brasil Florence Dravet, Universidade Católica de Brasília, Brasil Gelson Santana, Universidade Anhembi/Morumbi, Brasil Gislene da Silva, Universidade Federal de Santa Catarina, Brasil Guillermo Orozco Gómez, Universidad de Guadalajara Gustavo Daudt Fischer, Universidade do Vale do Rio dos Sinos, Brasil Hector Ospina, Universidad de Manizales, Colômbia Herom Vargas, Universidade Municipal de São Caetano do Sul, Brasil Inês Vitorino, Universidade Federal do Ceará, Brasil Jay David Bolter, Georgia Institute of Technology Jeder Silveira Janotti Junior, Universidade Federal de Pernambuco, Brasil John DH Downing, University of Texas at Austin, Estados Unidos José Afonso da Silva Junior, Universidade Federal de Pernambuco, Brasil José Carlos Rodrigues, Pontifícia Universidade Católica do Rio de Janeiro, Brasil José Luiz Aidar Prado, Pontifícia Universidade Católica de São Paulo, Brasil Kelly Cristina de Souza Prudêncio, Universidade Federal do Paraná, Brasil.
Laan Mendes Barros, Universidade Metodista de São Paulo, Brasil Lance Strate, Fordham University, USA, Estados Unidos Lorraine Leu, University of Bristol, Grã-Bretanha Lucia Leão, Pontifícia Universidade Católica de São Paulo, Brasil Malena Segura Contrera, Universidade Paulista, Brasil

Márcio de Vasconcellos Serelle, Pontifícia Universidade Católica de Minas Gerais, Brasil

Maria Aparecida Baccega, Universidade de São Paulo e Escola Superior de Propaganda e Marketing, Brasil

Maria Ataide Malcher, Universidade Federal do Pará, Brasil

Maria das Graças Pinto Coelho, Universidade Federal do Rio Grande do Norte, Brasil Maria Immacolata Vassallo de Lopes, Universidade de São Paulo, Brasil Maria Luiza Martins de Mendonça, Universidade Federal de Goiás, Brasi Mauro de Souza Ventura, Universidade Estadual Paulista, Brasil Mauro Pereira Porto, Tulane University, Estados Unidos Mirna Feitoza Pereira, Universidade Federal do Amazonas, Brasil Nilda Aparecida Jacks, Universidade Federal do Rio Grande do Sul, Brasil Osvando J. de Morais, Universidade de Sorocaba, Brasil Potiguara Mendes Silveira Jr, Universidade Federal de Juiz de Fora, Brasil Renato Cordeiro Gomes, Pontifícia Universidade Católica do Rio de Janeiro, Brasil Robert K Logan, University of Toronto, Canadá

Ronaldo George Helal, Universidade do Estado do Rio de Janeiro, Brasil Rose Melo Rocha, Escola Superior de Propaganda e Marketing, Brasil Rossana Reguillo, Instituto de Estudos Superiores do Ocidente, Mexico Rousiley Celi Moreira Maia, Universidade Federal de Minas Gerais, Brasil Sebastião Guilherme Albano da Costa, Universidade Federal do Rio Grande do Norte, Brasil

Simone Maria Andrade Pereira de Sá, Universidade Federal Fluminense, Brasil Tiago Quiroga Fausto Neto, Universidade de Brasília, Brasil Suzete Venturelli, Universidade de Brasília, Brasil Valerio Fuenzalida Fernández, Puc-Chile, Chile Veneza Mayora Ronsini, Universidade Federal de Santa Maria, Brasi Vera Regina Veiga França, Universidade Federal de Minas Gerais, Brasil

\section{COMISSÃO EDITORIAL}

Adriana Braga I Pontifícia Universidade Católica do Rio de Janeiro, Brasil Felipe Costa Trotta I Universidade Federal Fluminense, Brasil

\section{CONSULTORES AD HOC}

Ana Carolina Escosteguy, Pontifícia Universidade Católica do Rio Grande do Sul, Brasil Bruno Campanella, Universidade Federal Fluminense, Brasil

Edison Gastaldo, Universidade Federal Rural do Rio de Janeiro, Brasil Elizabeth Duarte, Universidade Federal de Santa Maria, Brasil Roseli Figaro, Universidade de São Paulo, Brasil

EDIÇ̃̃O DE TEXTO E RESUMOS I Susane Barros

SECRETÁRIA EXECUTIVA I Juliana Depiné

EDITORAÇÃo ELETRÔNICA I Roka Estúdio

TRADUÇ̃̃o I Sieni Campos e Markus Hediger
COMPÓS I www.compos.org.br

Associação Nacional dos Programas de Pós-Graduação em Comunicação

Presidente

Julio Pinto

Pontifícia Universidade Católica de Minas Gerais, Brasil juliopinto@pucminas.br

Vice-presidente

Itania Maria Mota Gomes

Universidade Federal da Bahia, Brasil

itania@ufba.br

Secretária-Geral

Inês Vitorino

Universidade Federal do Ceará, Brasil

inesvic@gmail.com 